\section{Effects of a Mindfulness Based Intervention on Psychological Distress and Romantic Relationships: Results of a Pilot Study}

\section{Dóra Vajda* and Enikö Csilla Kiss}

Department of Personality-and Health Psychology, Institute of Psychology, University of Pécs, Pécs, Hungary

\begin{abstract}
Various studies have demonstrated that mindfulness-based programs are related to positive psychological and physical outcomes. This pilot study was set out to investigate the relational and individual benefits of mindfulness training in well-functioning couples. In this randomized trial, we assigned 28 couples to the 10-week mindfulness training program intervention group ( $\mathrm{N}=14$ couples) or to a waitlist control group ( $\mathrm{N}=14$ couples). Relationship quality, relationship stress, and psychological distress were measured with standardized instruments. The results indicate the benefits of the meditation training. Couples in the treatment group showed significantly reduced psychological distress, somatization, hostility, psychoticism and paranoid ideation symptoms in comparison to couples who did not receive the intervention. The current pilot study provides preliminary support for reducing stress and enhancing psychological well-being in the sample of relatively well functioning couples. Despite encouraging findings, several limitations affect present study. Suggestions are given for future research based on better designed methodology and for future directions of investigation.

Keywords: Meditation; Mindfulness; Psychological distress; Relationship stress; Romantic relationships
\end{abstract}

\section{Introduction}

Mindfulness originates in Eastern Buddhist meditation traditions [1] and refers to pre-conceptual awareness, non-judgmentally acceptance of experience, purposeful control of attention and present-focused orientation [2,3]. Bishop et al., [4] defined mindfulness as "a process of regulating attention in order to bring a quality of non-elaborative awareness to current experience and a

*Corresponding author: Dóra Vajda, Department of Personality-and Health Psychology, Institute of Psychology, University of Pécs, Pécs, Hungary, Tel: +3672503600; E-mail: vajdadora@gmail.com

Citation: Vajda D, Kiss EC, (2016) Effects of a Mindfulness Based Intervention on Psychological Distress and Romantic Relationships: Results of a Pilot Study. J Community Med Public Health Care 3: 014.

Received: December 18, 2015; Accepted: March 24, 2016; Published: April 07,2016 quality of relating to one's experience within an orientation of curiosity, experiential openness, and acceptance (pg. 234)".

According to mindfulness theory, mindfulness is a basic human capacity and an ability that can be trained by practicing various forms of meditation [3]. By practicing mindfulness techniques, people seek to gain insight into patterns in their feelings, thoughts and interactions with others [5].

Research into mindfulness training has greatly expanded over the past several years. Various studies have demonstrated that mindfulness-based programs are related to positive psychological and physical outcomes [6,7]. The most frequently cited method of mindfulness training is the Mindfulness Based Stress Reduction (MBSR) program [6]. It was developed by Jon Kabat-Zinn in 1979. This program is designed to teach patients with chronic medical conditions how to live fuller, healthier, more adaptive lives [8]. The MBSR focuses on the cultivation of mindfulness through various activities (e.g., meditation, body scan and mindful yoga) and on the integration of this state into everyday life as a coping strategy for dealing with difficulties. The program has proven to be efficacious for clinical populations with a variety of physical and mental health issues including chronic pain, cancer, heart disease, depression and anxiety [5-7,9-12].

Furthermore, research suggests that MBSR is an effective intervention for psoriasis [13], fibromyalgia [14] and has been associated with significant decreases in general medical symptomatology [15]. The bulk of the evidence supports the benefits of MBSR in mentally and physically ill patients, whilst recently increasing attention focused on the benefits this practice might have on healthy people [16]. Studies on non-clinical populations show that mindfulness increases self-compassion, positive affection, quality of life and well-being and reduces rumination, somatization, anxiety, negative emotions, stress symptoms and aggression [16-21]. The MBSR intervention helped to reduce stress among nurses [22], therapists in training [23], and university students [18].

In a study, conducted by Astin [24], investigating the possibility of a standard course of MBSR to reduce stress in university undergraduates, a significantly higher reduction of stress from baseline, as measured by the global severity index of the Symptom Checklist 90-Revised (SCL-90-R), was observed in the MBSR group compared to the group on the waiting list.

Shapiro et al., [25] have demonstrated the beneficial effects of MBSR for medical students. Consistent with prior reports, the main findings of Greeson et al., [26] demonstrated the efficacy of a brief meditation-based mind-body workshop for students.

Recently, interest has grown in studying the role of mindfulness in romantic relationships [27,28]. Adapting the Mindfulness Based Stress Reduction [8]. Carson and his colleagues [27] developed an intervention program - Mindfulness Based Relationship Enhancement (MBRE) - for non-distressed couples. The MBRE was tested through a randomized controlled design with a waitlist control group. Forty-four couples participated in this program, which 
consist of an eight-week course with 150-minutes group sessions and a single daylong retreat. Mindfulness practice was facilitated through loving-kindness meditation, partner yoga, body-scan meditation and eye-gazing exercises. In this study mindfulness was mentioned as a way of living which is opened and nonjudgmental. The authors highlighted that mindfulness may lead to compassion for self and other which is especially relevant for enriching interpersonal functioning. Relative to wait-list controls ( $\mathrm{N}=22$ couples), couples who participated in the MBRE program ( $\mathrm{N}=22$ couples) scored significantly higher on relationship satisfaction, autonomy, relatedness, closeness and acceptance of partner at post-test as well as at a 3-month follow-up. The treatment group had significantly lower relational distress compared to the waitlist group. Individuals in the mindfulness treatment group were significantly higher in measures of optimism, spirituality, and relaxation and lower in psychological distress. Further analysis indicated that couples in mindfulness group improved in the areas of relationship happiness, were more resilient and increased ability to cope with stress [27]. The findings of this research emphasize the benefits of MBRE program which is efficacious in enriching current relationship functioning and improving individual psychological well-being.

It is important to note that recent research on meditation moved beyond self-reported outcomes to task-based and physiological measures of outcomes [29-31].

\section{The present study}

Summarizing the above literature indicates that mindfulness has proven to be effective and beneficial for a range of problems as well for clinical as for non-clinical populations $[3,11,12,17,21,32]$.

Although mindfulness based interventions are becoming more and more popular in Hungary, more studies are needed in order to confirm the benefits of mindfulness program for healthy population. We aimed to examine the feasibility and acceptability of mindfulness training in well-functioning, healthy couples. More specifically, the purpose of this study was to examine the effects of mindfulness based intervention on romantic relationships and individual well-being in a sample of university students and their partners. Based on the research of Carson et al., [27], we state that participating in meditation sessions would demonstrate benefits on measures of relationship variables as well as on measures of individual factors. Contrary to Carson and his colleague's research design, our study does not include couple centered mindful interventions.

It was hypothesized that participating in meditation sessions would (1) decrease overall psychological symptomatology measured by the SCL-90-R; (2) contribute to a decrease in relationship stress and (3) an increase in relationship quality.

\section{Methods}

\section{Participants and procedures}

The participants were recruited principally from university students and their partners at universities in Budapest, Hungary via advertisements, flyers and the internet. In total, 56 volunteers (28 couples) participated in the study. Exclusion criteria were (1) history of mental disorders (2) previous and current participation in regular yoga/meditation sessions. An inclusion criterion was (1) relationship existed for a period of at least six months. The age ranged from 20 to 38 for men (mean=25.42 years; $S D=4.00)$ and for women ranged from 19 to 33 ( mean= 24.17 years; $S D=3.26$ ).
Two couples were married, two were cohabiting, and twenty-four were in relationship (LAT: Living Apart Together). The length of current relationship ranged from 6 months to 8.5 years, with a mean of 3.025 years $(\mathrm{SD}=2.16)$. The intervention program was described as a challenging opportunity for couples to develop inner peace, mental balance and enrich new experience by participating in mindfulness sessions together with the partner.

Participants were randomized by a coin toss as a couple into the treatment group ( $\mathrm{N}=14$ couples) or waitlist control group $(\mathrm{N}=14$ couples). Following randomization, participants completed pretest surveys. The pre-intervention assessment occurred for all participants one week prior to the start of the first mindfulness session. Following the intervention, treatment group couples completed the same measures. The waitlist control group participants also completed their questionnaires at this same time. The post-intervention occurred after the last meditation session. Participating in the research was on a voluntary basis. Participants did not receive any compensation; there was no payment for taking part in the study. Data were collected between January and March 2014.

\section{Intervention}

The intervention was developed to train couples in mindfulness. The program included elements of MBSR, a relaxation and stress management program developed by Kabat-Zinn [8]. The intervention incorporated body scan, in which participants sequentially and non-judgmentally focus their attention on parts of the body and sitting meditation, in which participants focus their attention on their breathing, sounds in the environment, body sensations, and their stream of thoughts and emotions.

Members of the treatment group couples sat on the meditation pillow next to each other. Discussion of the study sessions occurred and feedback was provided as appropriate. The sessions were held in Meditation Center in Budapest and were facilitated by a psychologist trained in mindfulness-based interventions, as well as certified yoga instructor. The training was 2 hours in duration every second week for 10 weeks.

During the intervention, participating couples were required to practice 30 minutes formal daily meditation at home together with the partner. The psychologist who facilitated the trainings made audios (based on enrolled study conditions) for participants to use for guided mindfulness practice at home.

\section{Measures}

A questionnaire package was filled in before the first (pre-test) and after the last mindfulness session (post-test). The post-test comprised the same packet of questionnaire excluding demographics. A few more additional questions were asked in the post-test such as: participation in mindfulness sessions in Meditation Center, frequency of weekly meditation practice, and occurrence of any traumatic life events in the last two months.

\section{Sociodemographic questionnaire}

A self-designed measurement was used to obtain the demographic information of the participants. They were required to give their gender, age, grade, marital status and length of relationship. They also needed to report if they were receiving mindfulness training or medical treatment as these were considered as confounding variables.

Shortened Marital Stress Scale (SMSS) [33] is a 5 items self-report instruments designed to test relationship stress. Participants were 
asked to answer the questions (e.g., "Are there things you can't talk openly about with each other?") that required "yes" or "no" responses. Total scores are standardized to range from 0 to 5 , with higher scores (from 2 to 5) indicating greater marital stress and more relationship problems. Adequate reliability and validity of the scale have been demonstrated [33]. We found an alpha coefficient of 0.69 of the SMSS score.

Dyadic Adjustment Scale (DAS) [34]. This self-applied scale comprised of 32 Likert-type items, measuring relationship quality. The DAS assesses four facets of relationship adjustment: dyadic consensus, dyadic satisfaction, dyadic cohesion and affectional expression. A total score, derived by summing the scores on each of the four subscales, reflects overall adjustment. Possible scores range from 0 to 151, with higher scores indicating greater adjustment. For the purpose of this research only the overall scores of the DAS were utilized. Spanier [34] reported alpha coefficient for the Dyadic Adjustment Scale was excellent (0.96). We found an alpha coefficient of 0.75 for overall adjustment.

Symptom Checklist-90-Revised (SCL-90-R) is a 90-item self-report symptom inventory developed by Derogatis [35] to measure psychological symptoms and psychological distress. The SCL-90-R assesses psychological distress in terms of nine primary symptom dimensions: Somatization (SOM), Obsessive-Compulsive (OBS), Interpersonal Sensitivity (INT), Depression (DEP), Anxiety (ANX), Hostility (HOS), Phobic Anxiety (PHOB), Paranoid Ideation (PAR), and Psychoticism (PSY). Adequate reliability and validity have been demonstrated [35]. We found an alpha coefficient of 0.94 of SCL-90-R total score. The Cronbach's alpha coefficients varied from 0.72 to 0.92 for the subscales.

Alpha coefficients of all the questionnaires used in this study refer to the pre-test scores and were computed for overall sample ( $\mathrm{N}=28$ couples).

\section{Statistical analysis}

Analysis of Covariance (ANCOVA) was used to examine the change score from pretest to posttest while controlling for pretest scores. Men and women were analyzed separately to minimize collinearity issues within the couple data. This is an appropriate method of using ANCOVA with dyadic data when the two groups are made up of distinguishable members [36]. All data were analyzed by SPSS 21.0.

Partial eta-squared was used as the effect size measure. Partial eta-squared ( $\eta 2$ partial) is calculated by dividing the sum of squares of an effect by the addition of the sum of squares of the effect and the sum of squares of the error of that effect.

Cohen [37] suggested the following guidelines for effect size interpretation: partial $\eta^{2}=0.01$ is a small effect size, partial $\eta^{2}=0.06$ is a medium effect size and partial $\eta^{2}=0.14$ is a large effect size.

\section{Results}

On all measures no significant differences were found between treatment and control groups on pretest scores. No significant differences were found between treatment and control groups on demographic characteristics including age, length of relationship, marital status, educational attainment, and experience with mindfulness practice. Thus, there were comparable treatment and control groups in this study.
Results of ANCOVA for men showed improvement for those in the treatment group in total score of SCL-90-R, Somatization, Depression, Anxiety, Hostility, Phobic Anxiety, Paranoid Ideation and Psychoticism subscales when compared to the control. Men in the treatment group also had lower outcome score in relationship stress when compared to the control. Nonsignificant results were found for men in overall score of the Dyadic Adjustment Scale, Obsessive-Compulsive, and Interpersonal Sensitivity. Medium effect size with nonsignificant result for men was also found for Interpersonal Sensitivity (partial $\eta^{2}=0.12 ; \mathrm{p}=0.06$ ).

Women showed improvement for total score of SCL-90-R, and for Somatization, Obsessive-Compulsive, Hostility, Paranoid Ideation and Psychoticism subscales. Women had nonsignificant results for relationship stress, relationship quality, Interpersonal Sensitivity, Anxiety, Depression, and Phobic Anxiety. Medium effects with nonsignificant results were present for women in relationship quality (partial $\eta^{2}=0.07 ; \mathrm{p}=0.18$ ), and Anxiety (partial $\eta^{2}=0.07 ; \mathrm{p}=0.27$ ).

For complete results for men and women, see table 1 and 2.

\section{Discussion}

The results of the presented study demonstrated significantly reduced somatic and psychological symptoms, and decreased psychological distress in the sample of relatively well functioning couples following a 10 -week mindfulness training program. Men in the intervention group reported lower scores of marital stress, Anxiety, Depression, Phobic Anxiety, Psychoticism, Paranoid Ideation, Somatization, and Hostility when compared with the waitlist control group. Women in the treatment group indicated significantly better results in Somatization, Hostility, Psychoticism, Paranoid Ideation and Obsessive-Compulsive symptoms. These findings are consistent with a large body of literature that reported association between depression, anxiety, positive psychological outcomes and meditation $[7,38,39]$. As the outcome directions were the same for both genders, the differences in our results between men and women in the current study may be due to the low sample size. There are very few studies that have investigated the impact of gender on the effect of mindfulness-based intervention [27,40]. Future studies are needed to explore this issue in greater depth.

Our results indicated that men who participated in mindfulness training showed improvement in relationship stress. Research has shown that healthy romantic relationships are facilitated by individual psychological well-being [41] and mindfulness is positively associated with a number of potential positive intrapersonal factors for healthy relationships, such as positive affectivity, self-esteem, life satisfaction and inversely related to negative affectivity, neuroticism, depressive symptoms and stress reactivity [17].

According to this outcome, mindfulness training is recommended for men to be used for the reduction of relationship stress. Mindfulness - paying attention in the present moment, without judgment can help people break out of the negative reactions couples bring into their relationships. Mindfulness helps to better manage the body reactions, calms fears and anxiety, regulate emotions, which factors are all key ingredients for healthy, non-distressed relationships.

Although, an increase was detected in the mean score of dyadic adjustment when comparing pre- and post-intervention results in the intervention group, the analyses revealed insignificant outcomes. On one hand this may be due to the length and intensity of the current mindfulness training. In other words, presented mindfulness program 


\begin{tabular}{|c|c|c|c|c|c|c|c|c|c|c|c|}
\hline \multirow{3}{*}{ Measures } & \multicolumn{4}{|c|}{ Intervention group $(\mathrm{N}=14)$} & \multicolumn{4}{|c|}{ Control group $(\mathrm{N}=14)$} & \multirow{2}{*}{\multicolumn{3}{|c|}{ ANCOVA }} \\
\hline & \multicolumn{2}{|c|}{ Pretest } & \multicolumn{2}{|c|}{ Posttest } & \multicolumn{2}{|c|}{ Pretest } & \multicolumn{2}{|c|}{ Posttest } & & & \\
\hline & Mean & SD & Mean & SD & Mean & SD & Mean & SD & $F(1 ; 28)$ & $\mathrm{p}$ & Partial $\eta^{2}$ \\
\hline Shortened Marital Stress Scale & 0.28 & 0.46 & 0.07 & 0.26 & 0.35 & 0.49 & 0.42 & 0.51 & 5.68 & $0.02^{\star}$ & 0.18 \\
\hline Dyadic Adjustment Scale & 119.21 & 10.70 & 120.21 & 12.02 & 118.85 & 4.75 & 119.21 & 3.53 & 0.08 & 0.76 & 0.00 \\
\hline Symptom Checklist-90-R & 41.57 & 33.91 & 24.92 & 22.54 & 58.64 & 37.92 & 56.78 & 32.64 & 18.39 & $0.00^{* * *}$ & 0.42 \\
\hline Somatization & 4.07 & 4.76 & 1.92 & 2.49 & 6.85 & 5.70 & 7.00 & 5.76 & 17.29 & $0.00^{\star \star *}$ & 0.40 \\
\hline Obsessive-Compulsive & 7.07 & 5.55 & 6.57 & 5.50 & 9.50 & 5.25 & 9.07 & 5.07 & 0.31 & 0.58 & 0.01 \\
\hline Interpersonal Sensitivity & 4.57 & 4.25 & 3.07 & 2.99 & 6.00 & 7.50 & 5.50 & 5.89 & 3.64 & 0.06 & 0.12 \\
\hline Depression & 10.42 & 9.00 & 5.42 & 7.36 & 14.00 & 10.84 & 14.00 & 11.14 & 12.82 & $0.00^{* * *}$ & 0.34 \\
\hline Anxiety & 4.00 & 3.74 & 2.85 & 3.32 & 6.14 & 3.34 & 6.35 & 3.15 & 6.07 & $0.02^{*}$ & 0.19 \\
\hline Hostility & 3.78 & 4.94 & 1.92 & 2.36 & 5.42 & 2.87 & 4.85 & 1.95 & 17.37 & $0.00^{* * *}$ & 0.41 \\
\hline Phobic Anxiety & 1.07 & 2.05 & 0.35 & 0.84 & 1.35 & 1.49 & 1.28 & 1.38 & 6.63 & $0.01^{*}$ & 0.21 \\
\hline Paranoid Ideation & 3.64 & 3.65 & 1.57 & 1.65 & 3.78 & 4.59 & 3.85 & 3.86 & 16.16 & $0.00^{* * *}$ & 0.39 \\
\hline Psychoticism & 2.92 & 2.81 & 1.21 & 1.57 & 5.00 & 6.25 & 4.85 & 5.70 & 7.63 & $0.01^{\star}$ & 0.23 \\
\hline
\end{tabular}

Table 1: Results for Men.

Note: ${ }^{* *} p<0.001 ;{ }^{*} p<0.05$

\begin{tabular}{|c|c|c|c|c|c|c|c|c|c|c|c|}
\hline \multirow[t]{3}{*}{ Measures } & \multicolumn{4}{|c|}{ Intervention group $(\mathrm{N}=14)$} & \multicolumn{4}{|c|}{ Control group $(\mathrm{N}=14)$} & \multirow{2}{*}{\multicolumn{3}{|c|}{ ANCOVA }} \\
\hline & \multicolumn{2}{|c|}{ Pretest } & \multicolumn{2}{|c|}{ Posttest } & \multicolumn{2}{|c|}{ Pretest } & \multicolumn{2}{|c|}{ Posttest } & & & \\
\hline & Mean & SD & Mean & SD & Mean & SD & Mean & SD & $F(1 ; 28)$ & $\mathrm{p}$ & Partial $\eta^{2}$ \\
\hline Shortened Marital Stress Scale & 0.21 & 0.42 & 0.14 & 0.36 & 0.28 & 0.46 & 0.28 & 0.46 & 0.69 & 0.41 & 0.02 \\
\hline Dyadic Adjustment Scale & 123.28 & 5.01 & 124.42 & 8.71 & 118.64 & 9.09 & 117.92 & 6.78 & 1.88 & 0.18 & 0.07 \\
\hline Symptom Checklist-90-R & 48.71 & 28.04 & 30.85 & 23.60 & 60.28 & 39.04 & 56.64 & 33.53 & 6.03 & $0.02^{*}$ & 0.19 \\
\hline Obsessive-Compulsive & 7.64 & 5.19 & 4.21 & 4.24 & 10.85 & 7.68 & 10.85 & 7.71 & 12.06 & $0.00^{* * *}$ & 0.32 \\
\hline Interpersonal Sensitivity & 6.14 & 5.46 & 5.21 & 4.91 & 7.64 & 6.57 & 7.07 & 5.29 & 0.47 & 0.49 & 0.01 \\
\hline Depression & 11.78 & 6.99 & 8.64 & 7.48 & 15.92 & 10.90 & 14.64 & 10.32 & 1.52 & 0.22 & 0.05 \\
\hline Anxiety & 6.00 & 4.33 & 4.64 & 3.73 & 7.85 & 4.34 & 7.14 & 4.40 & 1.24 & 0.27 & 0.07 \\
\hline Hostility & 3.35 & 3.83 & 1.78 & 1.67 & 4.78 & 3.82 & 4.35 & 2.95 & 6.92 & $0.01^{*}$ & 0.21 \\
\hline Paranoid Ideation & 3.07 & 3.45 & 1.21 & 1.42 & 3.14 & 3.43 & 2.85 & 2.98 & 4.94 & $0.03^{*}$ & 0.16 \\
\hline Psychoticism & 3.21 & 4.66 & 1.07 & 1.63 & 3.28 & 4.00 & 3.07 & 2.89 & 9.72 & $0.00^{\star \star *}$ & 0.28 \\
\hline
\end{tabular}

Note: ${ }^{* * *} p<0.001 ;{ }^{*} p<0.05$

was not intense and long enough to contribute positive changes in relationship quality. On the other hand researchers [27] who carried out Mindfulness Based Relationship Enhancement program had couple centered mindful interventions, including loving-kindness meditation, body scan, eye-gazing exercises and such types of movements, which especially encourage collaborative practice between the couple. It should be noted, this mindfulness intervention of the presented study was mainly of not solely individual meditation, with training and home practice taking place with the partner.

Based on our data, we can conclude that in our research meditation training improved mostly intrapersonal outcomes but not interpersonal outcomes.

\section{Limitations}

While this pilot study provided feasibility of mindfulness intervention for couples and some interesting and valuable findings, there are limitations either. In order to analyze couples' data, multilevel analysis, the Actor-Partner interdependence model [42] would have been the most appropriate statistical techniques for measuring and testing dyadic data. It is essential that future research on treating couples take into account the multilevel nature of the data.

Although validated survey measures were used, self-report assessment is prone to social desirability bias and can fluctuate in reliability and validity. It is possible that non- specific factors such as social support, positive expectancy, and attention from an empathic instructor may have accounted, for some of the beneficial effects observed. The study might have a motivational bias of the selected participants. It can be assumed that mainly highly motivated couples volunteer to take part in the training and possibly more primed to focus on psychological and personal issues.

Subsequent studies are required to have an active control group in the sense that they were oriented to controlling for nonspecific effects of the intervention group (e.g., social support, demand characteristics and expectancy effects). Pilot studies often have to cope with small sample sizes which limit their scope to evaluate the effects of intervention. Future studies can test the different effects of 
Citation: Vajda D, Kiss EC, (2016) Effects of a Mindfulness Based Intervention on Psychological Distress and Romantic Relationships: Results of a Pilot Study. J Community Med Public Health Care 3: 014.

Mindfulness Based Stress Reduction and any other active groups (e.g., relaxation, loving-kindness meditation) to determine which intervention would be the most beneficial for the different groups or particular types of couples (e.g., couples undergoing infertility counseling, couples with chronic illness).

Future research could include methods of measuring trait mindfulness, and additional forms of assessment that extend and strengthen the self-report measures (e.g., improved diary collection procedures, interviews). Finally, studies could also benefit from including follow-up assessment to determine whether the benefits of mindfulness training are enduring.

\section{References}

1. Baer RA, Smith GT, Allen KB (2004) Assessment of mindfulness by self-report: the Kentucky inventory of mindfulness skills. Assessment 11: 191-206.

2. Brown KW, Ryan RM, Creswell JD (2007) Mindfulness: Theoretical Foundations and Evidence for its Salutary Effects. Psychological Inquiry 18: 211-237.

3. Kabat-Zinn J (2003) Mindfulness-Based Interventions in Context: Past, Present, and Future. Clinical Psychology: Science and Practice 10: 144-156.

4. Bishop SR, Lau M, Shapiro S, Carlson L, Anderson N, et al. (2004) Mindfulness: A proposed Operational Definition. Clinical Psychology: Science and Practice 11: 230-242.

5. Teasdale JD, Segal ZV, Williams JM, Ridgeway VA, Soulsby JM, et al. (2000) Prevention of relapse/recurrence in major depression by mindfulness-based cognitive therapy. J Consult Clin Psychol 68: 615-623.

6. Baer RA (2003) Mindfulness Training as a Clinical Intervention: A Conceptual and Empirical Review. Clinical Psychology: Science and Practice 10: 125143.

7. Grossman Pac, Niemann L, Schmidt S, Walach H (2004) Mindfulness-based stress reduction and health benefits. A meta-analysis. J Psychosom Res 57 $35-43$.

8. Kabat-Zinn J (1982) An outpatient program in behavioral medicine for chronic pain patients based on the practice of mindfulness meditation: theoretical considerations and preliminary results. Gen Hosp Psychiatry 4: 33-47.

9. Carlson LE, Brown KW (2005) Validation of the Mindful Attention Awareness Scale in a cancer population. J Psychosom Res 58: 29-33.

10. Irving JA, Dobkin PL, Park J (2009) Cultivating mindfulness in health care professionals: a review of empirical studies of Mindfulness-Based Stress Reduction (MBSR). Complement Ther Clin Pract 15: 61-66.

11. Segal Z, Williams M, Teasdale J (2002) Mindfulness-based cognitive therapy for depression: A new approach to preventing relapse. Guilford Press, New York, USA.

12. Strauss C, Cavanagh K, Oliver A, Pettman D (2014) Mindfulness-Based Interventions for People Diagnosed with a Current Episode of an Anxiety or Depressive Disorder: A Meta-Analysis of Randomised Controlled Trials. PLoS One 9: 96-110.

13. Kabat-Zinn J, Wheeler E, Light T, Skillings A, Scharf MJ, et al. (1998) Influence of a mindfulness meditation-based stress reduction intervention on rates of skin clearing in patients with moderate to severe psoriasis undergoing phototherapy (UVB) and Photochemotherapy (PUVA). Psychosom Med 60: $625-632$

14. Kaplan KH, Goldenberg DL, Galvin-Nadeau M (1993) The impact of a meditation-based stress reduction program on fibromyalgia. Gen Hosp Psychiatry 15: 284-289.

15. Kabat-Zinn J, Massion AO, Kristeller J, Peterson LG, Fletcher KE, et al. (1992) Effectiveness of a meditation-based stress reduction program in the treatment of anxiety disorders. Am J Psychiatry 149: 936-943.
16. Chiesa A, Serretti A (2009) Mindfulness-based stress reduction for stress management in healthy people: a review and meta-analysis. J Altern Complement Med 15: 593-600.

17. Brown KW, Ryan RM (2003) The benefits of being present: mindfulness and its role in psychological well-being. J Pers Soc Psychol 84: 822-848.

18. Shapiro SL, Oman D, Thoresen CE, Plante TG, Flinders T (2008) Cultivating mindfulness: effects on well-being. J Clin Psychol 64: 840-862.

19. Khoury B, Lecomte T, Fortin G, Masse M, Therien P, et al. (2013) Mindfulness-based therapy: a comprehensive meta-analysis. Clin Psychol Rev 33: 763-771.

20. Gu J, Strauss C, Bond R, Cavanagh K (2015) How do mindfulness-based cognitive therapy and mindfulness-based stress reduction improve mental health and wellbeing? A systematic review and meta-analysis of mediation studies. Clin Psychol Rev 37: 1-12.

21. Lamothe M, Rondeau É, Malboeuf-Hurtubise C, Duval M, Sultan S (2016) Outcomes of MBSR or MBSR-based interventions in health care providers: A systematic review with a focus on empathy and emotional competencies. Ther Med 24: 19-28.

22. Cohen-Katz J (2004) Mindfulness-based stress reduction and family systems medicine: A natural fit. Families, Systems, \& Health 22: 204-206.

23. Shapiro SL, Brown WK, Biegel GM (2007) Teaching Self-Care to Caregivers: Effects of Mindfulness-Based Stress Reduction on the Mental Health of Therapists in Training. Training and Education in Professional Psychology 1: $105-115$

24. Astin JA (1997) Stress reduction through mindfulness meditation. Effects on psychological symptomatology, sense of control, and spiritual experiences. Psychother Psychosom 66: 97-106.

25. Shapiro SL, Schwartz GE, Bonner G (1998) Effects of mindfulness-based stress reduction on medical and premedical students. J Behav Med 21: 581 599.

26. Greeson JM, Toohey MJ, Pearce MJ (2015) An adapted, four-week mindbody skills group for medical students: reducing stress, increasing mindfulness, and enhancing self-care. Explore (NY) 11: 186-192.

27. Carson JW, Carson KM, Gil KM, Baucom DH (2007) Self-expansion as a mediator of relationship improvements in a mindfulness intervention. J Marital Fam Ther 33: 517-528.

28. Christensen A, Sevier M, Simpson LE, Gattis K (2004) Acceptance, mindfulness, and change in couple therapy. In: Steven $C$ Hayes, Victoria M Follette, Marsha M Linehan (eds.). Mindfulness and acceptance: Expanding the cognitive behavioral tradition. Guilford Press, New York, USA.

29. Creswell JD, Pacilio LE, Lindsay EK, Brown KW (2014) Brief mindfulness meditation training alters psychological and neuroendocrine responses to social evaluative stress. Psychoneuroendocrinology 44: 1-12.

30. Kemeny ME, Foltz C, Cavanagh JF, Cullen M, Giese-Davis J, et al. (2012) Contemplative/emotion training reduces negative emotional behavior and promotes prosocial responses. Emotion 12: 338-350.

31. Turan B, Foltz C, Cavanagh J, Wallace BA, Cullen M, et al. (2015) Anticipatory Sensitization to Repeated Stressors: The Role of Initial Cortisol Reactivity and Meditation/emotion Skills Training. Psychoneuroendocrinology 52: 229238

32. Bishop SR (2002) What do we really know about mindfulness-based stress reduction? Psychosom Med 64: 71-83.

33. Balog P, Székely A, Szabó G, Kopp M (2006) The psychometric properties of the Shortened Marital Stress Scale. J Ment Health Psychosom 7: 193-203.

34. Daspe MĖ, Sabourin S, Péloquin K, Lussier Y, Wright J (2013) Curvilinear associations between neuroticism and dyadic adjustment in treatment-seeking couples. J Fam Psychol 27: 232-241. 
Citation: Vajda D, Kiss EC, (2016) Effects of a Mindfulness Based Intervention on Psychological Distress and Romantic Relationships: Results of a Pilot Study. J Community Med Public Health Care 3: 014.

35. Möller-Leimkühler AM, Mädger F (2011) Personality factors and mental health outcome in caregivers of first hospitalized schizophrenic and depressed patients: 2-year follow-up results. Eur Arch Psychiatry Clin Neurosci 261: $165-172$.

36. DeCoster J (2002) Using ANOVA to examine data from groups and dyads. Tuscaloosa.

37. Parker R, Hagan-Burke S (2007) Useful effect size interpretations for single case research. Behav Ther 38: 95-105.

38. Hofmann SG, Sawyer AT, Witt AA, Oh D (2010) The effect of mindfulness-based therapy on anxiety and depression: A meta-analytic review. J Consult Clin Psychol 78: 169-183.
39. Majumdar M, Grossman P, Dietz-Waschkowski B, Kersig S, Walach H (2002) Does mindfulness meditation contribute to health? Outcome evaluation of a German sample. J Altern Complement Med 8: 719-730.

40. de Vibe M, Solhaug I, Tyssen R, Friborg O, Rosenvinge JH, et al. (2013) Mindfulness training for stress management: a randomised controlled study of medical and psychology students. BMC Med Educ 13: 107.

41. Epstein NB, Baucom DH (2002) Enhanced cognitive-behavioral therapy for couples: A contextual approach. American Psychological Association, Washington DC, USA.

42. Kenny DA, Kashy DA, Cook WL (2006) Dyadic data analysis. Guilford Press New York, USA. 